Revue d'études américaines. American Studies Journal

$2 \mid 2017$

(Hi)stories of American Women: Writings and Rewritings / Call and Answer: Dialoguing the American West in France

\title{
Philip Roth, Romans et nouvelles (1959-1977)
}

\section{Arnaud Schmitt}

\section{OpenEdition}

\section{Journals}

Édition électronique

URL : https://journals.openedition.org/transatlantica/10576

DOI : $10.4000 /$ transatlantica. 10576

ISSN : 1765-2766

Éditeur

Association française d'Etudes Américaines (AFEA)

Référence électronique

Arnaud Schmitt, "Philip Roth, Romans et nouvelles (1959-1977) », Transatlantica [En ligne], 2 | 2017, mis en ligne le 19 avril 2019, consulté le 02 février 2023. URL : http://journals.openedition.org/ transatlantica/10576 ; DOI : https://doi.org/10.4000/transatlantica.10576

Ce document a été généré automatiquement le 2 février 2023

\section{c) (i) $९$}

Creative Commons - Attribution - Pas d'Utilisation Commerciale - Pas de Modification 4.0 International - CC BY-NC-ND 4.0

https://creativecommons.org/licenses/by-nc-nd/4.0/ 


\title{
Philip Roth, Romans et nouvelles (1959-1977)
}

\author{
Arnaud Schmitt
}

\section{RÉFÉRENCE}

Philip Roth, Romans et nouvelles (1959-1977). Préface par Philippe Jaworski, Édition établie par Brigitte Félix, Aurélie Guillain, Paule Lévy et Ada Savin, Paris : La Pléiade, Gallimard, 2017, 1280 pages, $69,90 €$, ISBN-10 : 2070196828

1 Philip Roth entre donc dans la Pléiade, suivant la logique qui a vu la publication de l'intégralité de ses textes dans la Library of America débuter dès 2005, à savoir une «panthéonisation» qui identifie son œuvre comme l'une des plus importantes de la littérature américaine post-Seconde Guerre mondiale. Mais ce processus de reconnaissance officielle a débuté dès les années 80 et 90 avec l'attribution de nombreux prix littéraires prestigieux, aux États-Unis et dans le reste du monde. Et pourtant, comme cela est mentionné à de nombreuses occasions dans ce très beau volume, Roth fut un auteur par qui le scandale arriva. Cependant son immense talent finit par prendre le pas sur le scandale, du moins dans la deuxième partie de sa carrière. Et puis ainsi va l'histoire littéraire : les textes scandaleux d'hier deviennent les normes d'aujourd'hui, même si certains romans de Roth conservent une puissance déstabilisatrice.

2 Ce premier volume comprend cinq textes, dans l'ordre chronologique de parution : un recueil de nouvelles, Goodbye, Columbus (1959), et quatre romans : La Plainte de Portnoy (1969), Le Sein (1972), Ma vie d'homme (1974) et Professeur de désir (1977). Durant cette même période, quatre autres romans furent publiés par Roth mais ne figurent pas dans le présent volume car, selon Paule Lévy, ils sont "plus laborieux » (elle fait référence à Laisser Courir [1962] et Quand elle était gentille [1967], « son roman sans Juifs » [xl-xli]). Les deux autres textes n'ayant pas été « retenus » (lxxi) sont Tricard Dixon et ses copains (1971) et Le Grand Roman américain (1973). Il est toujours délicat de parler de textes 
majeurs et mineurs lorsque l'ensemble de l'œuvre d'un auteur aussi important que Philip Roth est évalué, tant les textes mineurs semblent alimenter ou préparer les textes majeurs, surtout dans le cadre d'une approche génétique, mais il serait cependant difficile de contester les choix qui ont été effectués ici. De tout façon, à l'exception peut-être de Portnoy, et en se basant sur la réception critique des textes ou les prix reçus, les grands romans de Philip Roth ont tous été publiés après 1977. On peut toutefois imaginer qu'il sera plus délicat dans les volumes à venir d'exclure certains textes, tant les années 80 et 90 , et même le début du nouveau siècle (jusqu'à la publication The Plot against America peut-être), ont vu l'auteur américain atteindre des sommets créatifs, proposant une succession de ce que nous pourrions appeler, pour ceux qui n'ont pas renoncé à utiliser ce type d'étiquettes hautement subjectives, des chefs-d'œuvre. Mais comme cela est souvent le cas dans le processus complexe de la création littéraire, avant les chefs-d'œuvre viennent, non pas les brouillons, mais les romans d'apprentissage (dans le cas des textes présentés ici, à la fois pour l'auteur et les personnages).

3 En dehors de tout l'appareil critique dont nous allons parler plus bas, ce volume propose aussi un travail de profondes révisions des traductions existantes (par Ada Savin, Paule Lévy, Brigitte Félix et Aurélie Guillain), « [c]onformément à la volonté de l'auteur », et cela afin de "rendre au plus près l'oralité des textes » (lxxi). Il est toujours peu évident d'évaluer non pas la qualité de la traduction mais bien sa fidélité au texte original, et n'étant pas traducteur, je ne m'y risquerai pas. Il faut pourtant noter que ces traductions révisées semblent en effet mettre l'accent sur les signes multiples d'oralité et d'irrévérence qui caractérisent la « langue rothienne » dans cette première partie de son œuvre. Les romans de ce que nous pourrions appeler la maturité, même si Roth devint un auteur mature très jeune, afficheront un équilibre presque savant entre une langue incroyablement construite, voire soutenue, et cette spontanéité orale qui innerve Portnoy de bout en bout. La recette fut élaborée par Saul Bellow dans The Adventures of Augie March, texte fondateur de ce qu'on appellera par la suite la littérature juive américaine ${ }^{1}$ (et dont Bellow, Roth et Malamud représentent pour beaucoup les trois pères fondateurs).

4 Comme cela est toujours le cas pour La Pléiade, l'appareil critique est conséquent et s'articule autour d'une préface (de Philippe Jaworski), d'une introduction (de Paule Lévy), d'une "chronologie » (à la fois biographique et bibliographique), d'une notice pour chaque texte (notices rédigées respectivement par Ada Savin, Paule Lévy, Brigitte Félix, Aurélie Guillain et de nouveau Brigitte Félix). Un nombre important de notes figure dans le recueil, afin de contextualiser et expliciter les nombreuses références culturelles, intertextuelles, linguistiques (un glossaire des mots yiddish et hébreux se trouve en fin de volume), historiques ou encore sociologiques. Ce travail d'élaboration des notes a été accompli avec minutie et sa lecture permet de se rendre compte combien l'idée de contemporanéité est relative. En effet, un texte publié en 1959 est déjà, par ses références sociologiques par exemple, fortement défamiliarisant (même lorsqu'on possède une très bonne connaissance du contexte américain). Enfin, le volume se clôt sur une bibliographie des textes de Roth et des principales études critiques consacrées à son œuvre.

5 Même si le meilleur reste à venir dans l'œuvre de Roth comme cela a déjà été précisé, les grands thèmes fondateurs et structurants du récit rothien sont présents dès Goodbye, Columbus. Dans sa préface, Philippe Jaworski les énumère et les explicite de 
manière concise mais précise: l'homo scriptor (n'oublions jamais que les grands narrateurs rothiens sont presque tous écrivains, Zuckerman, vecteur de tant de sèmes biographiques inhérents à Roth, en étant l'archétype), la "dimension de la dérision " (x) et les « pitres » (xiv), le corps (xxvi, Brigitte Félix souligne elle aussi l'importance de la «corporalité » chez Roth [1128]) et la mort (xxviii), la judéité mais aussi l'américanité, et son corollaire, la famille, ou plus exactement un «jeu de massacre familial» (xii); ajoutons néanmoins que cette étiquette d'auteur juif américain a toujours été problématique pour Roth ${ }^{2}$ : «Updike, for example, is an American writer whose territory often is small-town Pennsylvania and New England, and I'm an American writer whose territory is a small section of New York » (Kauvar 296). L'auteur n'est cependant pas toujours le mieux placé pour parler de lui-même ou de son œuvre, et les masques dont il affuble ses personnages, Zuckerman en tête, n'ont certainement pas pour fonction de clarifier les choses, ce qui serait de toute façon contraire à tout son projet littéraire. Roth a eu très tôt l'ambition de poser problème (dans et de par son œuvre), particulièrement par cet aspect : «la vie supposée cachée de l'artiste, voilà ce qui fascine, jusqu'à la folie, les biographes, les critiques, les lecteurs » (xxi) et Roth, même s'il a toujours prétendu le contraire, a tout fait pour alimenter cette " folie ». En effet, si « [d]errière Kepesh, les critiques cherchent Roth » (1165), ils continueront à le faire avec Zuckerman, un personnage/narrateur encore plus aligné sur la vie de son auteur empirique.

6 L'introduction de Paule Lévy reprend certains de ces thèmes, mais explore principalement la piste de la genèse et du contexte dans lequel l'œuvre a émergé, avant de présenter chaque texte figurant dans ce volume. Elle affirme que « [t]out est déjà là, et c'est à cette époque et avec ces ouvrages qu'en vérité Philip Roth devient Philip Roth ", et confirme que les contours thématiques de l'œuvre rothienne sont tracés dès ses premiers textes. Mais elle démontre que cette transformation ne s'est pas faite dans la quiétude. En effet si sa fiction regorge de conflits, la réception de ses textes en a, à son tour, suscité de nombreux. Paule Lévy revient sur certains de ces épisodes marquants qui ont suivi la sortie de, principalement, Goodbye, Columbus et Portnoy. Dans le premier cas, même si Roth " reçoit les éloges appuyés de Saul Bellow, Alfred Kazin, Irving Howe et Leslie Fiedler, ces 'quatre tigres de la littérature juive américaine' " (xxxix), le livre "soulève un tollé dans les milieux conservateurs juifs » (xxxix), qui accompagnera Roth durant de longues années jusqu'à « l'explosion » (1101) Portnoy, un phénomène littéraire "imprégné du climat subversif des sixties » (xlv) qui apporta à son auteur un succès mêlé d'indignation. La sortie de Portnoy demeure l'événement incontournable de cette première partie de l'œuvre, et représente peutêtre aussi la pierre angulaire (stylistiquement parlant) de l'ensemble de son œuvre. Il est bon de rappeler, notamment pour les plus jeunes lecteurs de Roth, le tumulte causé par cette première partie d'une œuvre qui, pourtant, comme Paule Lévy le rappelle de très belle manière dans sa conclusion, "célèbre dans sa verdeur, sa fougue, son anticonformisme jubilatoire et 'sa merveilleuse capacité à sentir la complexité des choses' [...], l'extraordinaire vitalité du processus créateur, l'inépuisable richesse de l'art et de la vie » (liii). Mais Roth n'a eu de cesse de susciter les polémiques. Et même si la voix de ses détracteurs se fit moins intense par la suite (ou la qualité de sa fiction tendit à l'étouffer), il faut garder à l'esprit l'omniprésence de la figure de la Némésis chez Roth, à la fois dans sa matière diégétique, comme le démontre Aurélie Guillain (1133) dans la notice très complète qu'elle consacre à Ma vie d'homme, et également dans la réception de ses textes. Ce thème est récurrent dans tout l'appareil critique proposé 
dans ce volume, et on ne peut que s'en féliciter, tant Roth s'est nourri de cette réception problématique en la réinsérant dans sa propre fiction: "Par le biais d'une ironique 'mise en abyme', dans les années 1980 Roth fera du conflit qui l'a opposé à son public l'objet même de la tétralogie Zuckerman enchaîné » (xlvi).

7 Chaque notice propose donc, entre autres, une approche génétique et démontre une remarquable connaissance des conditions de création de ces différents textes. L'ensemble, même pour un spécialiste de Roth, révèle des pans peu connus de l'histoire de cette œuvre. Par exemple, Ada Savin, dans la notice consacrée à Portnoy, nous apprend que ce roman «fut en fait construit 'sur les ruines de quatre projets abandonnés', auxquels il [Roth] avait 'consacré beaucoup de travail' entre 1962 et 1967 » (1087), passionnants tâtonnements qui n'empêchèrent pas Roth in fine de mener à terme l'écriture de ce roman monstre qui représente avant tout, comme Ada Savin le fait très justement remarquer, « un tour de force formel » (1093). De la même manière, les notices d'Aurélie Guillain et de Brigitte Félix consacrées respectivement à Ma vie d'homme et Professeur de désir mettent en avant la façon dont ces deux romans se sont mutuellement alimentés, Roth étant probablement à l'époque à la recherche non pas d'une forme (elle continuera à changer), mais bien d'un narrateur à qui il confiera plus tard la barre de nombreux romans. Plus que tout autre texte, Ma vie d'homme pose les prémisses du tournant postmoderne (que Roth, clairement dans le déni, a toujours refusé d'admettre) pris par son œuvre dans les années 80 (avec La Contrevie en point d'orgue). Aurélie Guillain met justement l'accent sur l'importance de la métalepse dans ce roman, trope qui reviendra sous plusieurs formes (notamment celle de l'auteur) dans ses romans suivants. Et si Zuckerman n'y tient qu'un rôle secondaire, on sait que le narrateur premier, Tarnopol, va disparaitre pour laisser la place pendant près de trois décennies (en comptant quelques interruptions) à Nathan Zuckerman, personnage et narrateur de second rang en 1977. Et si selon Aurélie Guillain, « Ma vie d'homme est une apologie de la littérature " (1145), la tétralogie Zuckerman, dont la publication commencera en 1979, en représentera l'un des sommets. On ne peut donc que se réjouir de la publication (prochaine?) du second volume, en particulier si la qualité de l'appareil critique demeure à la hauteur de celle proposée dans ce premier volume.

\section{BIBLIOGRAPHIE}

\section{đEUVRES CITÉES}

- KAUVAR, Elaine M., « This Doubly Reflected Communication: Philip Roth's ‘Autobiography’" , Contemporary Literature, Fall, 1995.

- PODHORETZ, Norman. «Philip Roth: The Great American Novelist », The American Spectator, September 1981. 


\section{NOTES}

1. Paule Lévy rappelle l'influence que le roman de Bellow a eue sur Roth lui-même et qui fut pour ce dernier « une révélation » (xliv).

2. "To be sure, all three of these writers [Bellow, Malamud, Roth], and especially Bellow, would express resentment when they were identified as Jewish writers, as though this diminished them and made their work less American or less universal" (Podhoretz 26).

INDEX

Thèmes : Recensions

\section{AUTEURS}

ARNAUD SCHMITT

Université de Bordeaux - EA CLIMAS Bordeaux Montaigne 